\title{
Development, Gender and Sport: Theorizing a Feminist Practice of the Capabilities Approach in Sport for Development
}

\author{
Sarah Zipp \\ University of Stirling \\ Tavis Smith and Simon Darnell \\ University of Toronto
}

\begin{abstract}
Sport for development (SFD) research and practice has become more critically examined recently, with many scholars calling for better understanding of how and why sport might contribute to the global development movement. Developing and refining theoretical approaches is key to unpacking the complexities of SFD. Yet, theory development in SFD is still relatively young and often relies on oversimplified theory of change models. In this article, the authors propose a new theoretical approach, drawing upon the capabilities approach and critical feminist perspectives. The authors contend that the capabilities approach is effective in challenging neoliberal ideologies and examining a range of factors that influence people's lived experiences. They have woven a "gender lens" across the capabilities approach framework, as feminist perspectives are often overlooked, subjugated, or misunderstood. The authors also provide an adaptable diagrammatic model to support researchers and practitioners in applying this framework in the SFD context.
\end{abstract}

Keywords: development through sport, economic development, female athletes, feminism, social development

Recently, two trends have emerged within the theorizing and assessment of sport for development (SFD): a critical feminist approach, which examines how gender is experienced in SFD, questioning traditional SFD approaches that may unwittingly reinforce restrictive gender roles (Carney \& Chawansky, 2016; McDonald, 2015; Saavedra, 2009); and the application of the capability approach (CA), which considers the extent to which capabilities might offer an appropriate, meaningful, and effective basis from which to understand development through sport (Darnell \& Dao, 2017; Suzuki, 2017; Svensson \& Levine, 2017).

In this study, we aim to combine these two trends within a single theoretical and practical framework, one that proposes the $\mathrm{CA}$ as the basis of a committed and refined feminist approach to SFD and provides a diagrammatic model for practical application. First, the CA seeks to understand the ways that SFD participants experience SFD programming, and the ways in which those experiences reflect the real opportunities, mitigated by gender, that people have lives that they value. Second, the CA provides key conceptual tools - capabilities, conversion factors, and adaptive preferences-to facilitate a critical feminist engagement with the ways that these experiences are mediated by the social, cultural, economic, and political environments. We suggest that although the CA has been used to good effect in recent SFD theorizing and research, none of this work has focused on the question of gender or its place within a feminist approach to SFD.

This is a significant gap or oversight, leaving the concept of gender underdeveloped within SFD research. For example, Thorpe and Chawansky call on sport managers to recognize "women's lived experiences as a valid and valuable form of knowledge that could be used to inform management approaches adopted by SFD organizations" (2017, p. 558). Their call points to the need for, and benefits of, a practical framework which recognizes feminist

Zipp is with the University of Stirling, Stirling, United Kingdom. Smith and Darnell are with the University of Toronto, Toronto, Ontario, Canada. Zipp (sarah.zipp@ stir.ac.uk) is corresponding author. struggles amidst patriarchal systems and environments, although also seeing women's experiences as strengths that are useful toward achieving positive development outcomes.

To demonstrate how girls' and women's "lived experiences" are overlooked or marginalized in SFD, we present an illustrative example to SFD research: menstruation. Menstruation, menstrual health education, and menstrual hygiene management are almost entirely absent in the SFD literature, despite the fact that these concerns directly impact the lives of all or most female SFD participants (Harrison, 2018). Meanwhile, these topics have been researched by feminist scholars in development studies for decades and a recent movement has brought menstruation to the forefront of gender in international development research and practice (Bobel, 2018). We expand on this example later in this article, along with other examples of how adaptive preferences help illustrate often overlooked aspects of gendered socialization into sport.

We contend that these critical feminist perspectives must be woven into all SFD theory, and that the CA model provided can help researchers and practitioners "bridge the gap" to better understand gender and SFD. To build this case, the remainder of the article proceeds in four parts. In the next section, we present our conceptualization of critical issues at the intersection of gender, development, and SFD. This leads into a discussion of the CA within SFD and presents our diagrammatic model, ${ }^{1}$ examining the key elements of capabilities, conversion factors, and adaptive preferences. We then discuss the specific ways in which we see the CA model making a contribution to advancing a feminist approach to SFD, demonstrating how the model includes overlooked concerns such as menstruation and adaptive preferences. Finally, the article concludes with a summary of the main points as well as questions for future theorizing and research in this area.

\section{Gender, Development, and SFD}

Gender is complex within any social context, but in the field of SFD, gender formations and perspectives are further complicated by the various histories and cultures of sport that have tended to 
place restrictions on the full participation of girls and women. It is these kinds of cultures and histories that have led feminist researchers broadly to call for critical understandings of gender, and to seek understandings of the ways in which gender is performed within social structures and formations. Following Knoppers and Anthonissen, "Doing gender consists of engaging in actions that are part of social processes. These actions include practices of power that support or challenge domination of men and subordination of women ..." (2008, p. 94). From this perspective, gender is a social construct and situated in social practices and processes, such as divisions of labor and notions of sexuality. Gender also intersects with various aspects of social identity, such as class, race, and sexuality that construct the lived experience of SFD participants and practitioners (Oxford \& McLachlan, 2018; Oxford \& Spaaij, 2019). This feminist perspective leads to critical questions regarding gendered divisions of labor in sport, and the ways these divisions have been, and still are, generated, including within the policies and practices of SFD. ${ }^{2}$

For the purposes of this study, we view gender as fluid, relational, and nonbinary; all the while attached to both symbolic and real power that still tends to privilege men and subjugate women. This dynamic plays out at the personal level, but also through social, cultural, political, and economic power at broader scales, which perpetuate many of the gendered inequalities that SFD initiatives strive to address. For these reasons, we concur with calls to apply a feminist lens to theory development within SFD. One such call, from Sarah Oxford and Fiona McLachlan (2018), encourages SFD researchers to "look at the limits of postfeminist, neoliberal, or postmodern thinking and theorizing that ignores or rejects history and structure."

In response, we consider how broader gender norms often constrain engagement in sport and that the dynamics of development initiatives are gendered through larger macrolevel factors such as economic policies, education systems, and postcolonial legacies (Chawansky \& Hayhurst, 2015; Hayhurst, Kay, \& Chawansky, 2015). The key point here is to approach gender (and girls and women) less as the targets of SFD, and instead to view gender more as a fundamental aspect of the landscape of development inequalities broadly, and the experiences of SFD stakeholders and participants specifically. Approaching gender in SFD in this way, we contend, is still relatively novel, given the propensity of theoretical frameworks and models in SFD that aim to compartmentalize or isolate gender, or consider it to be a distinct thematic area of the broader SFD field (see Zipp \& Nauright, 2018).

In turn, a feminist approach to SFD also calls into question the ways in which young people in general, and girls and young women in particular, are still seen as those to be helped (or even saved) through sport-based initiatives or interventions. Indeed, young people in need of help and improvement remain nearly fundamental to the policies, practices, politics, and preferred narratives of SFD (Darnell, 2007, 2012). SFD's beneficiaries or user groups are often portrayed as vulnerable and/or deficient, and this is particularly the case for girls and young women (Forde \& Frisby, 2015; Zipp \& Nauright, 2018). The "girling" of development or positioning the empowerment of vulnerable girls as a key to improving communities and societies, is an emerging phenomenon in SFD and the broader development field (Bobel, 2018; Hayhurst, 2011, p. 532). In this way, gender intersects with neoliberal ideological narratives, where poor, often non-White, people are in need of help from the industrialized West. Girls and women are viewed as needing "to be empowered" so that they might better navigate their environments and overcome their marginalization (McDonald, 2015; Oxford \& McLachlan, 2018).

Although these complex issues surrounding how to approach gender in development (GID) have been discussed in the broader field of development studies for decades, this type of third wave, critical feminist approach is relatively new to SFD (Chawansky, 2011). In development studies, simply inserting women and girls into existing development structures and systems has been labeled the gender in development approach, and feminist researchers have criticized GID as ineffective and discriminatory. Esther Boserup's 1970 text, Women's Role in Economic Development, was one of the first to question the ostensibly gender-neutral approach to mainstream development economics. Her work set the stage for the emergence of the gender and development (GAD) paradigm, which called for more examination, and transformation, of the social and economic systems, structures, and processes that maintain gender hierarchies.

We contend that, overall, much of what constitutes current SFD policies and practices illustrate minimal understanding, appreciation, or implementation of the insights of the GAD perspective. That is, although many SFD programs are designed with the goal of supporting gender equality, they remain largely rooted in gender binaries and heteronormative ideologies, and/or in integrating girls and women into masculine norms and cultures. For example, most coaches and leaders of SFD programs are men and the general lack of female role models in SFD can marginalize girls and women (Meier, 2015). Further, SFD is still often built upon historically masculine sports, such as football/soccer or cricket. Although it is understandable that SFD programs would choose sports with wide appeal, girls and women are often less likely to have had the opportunity to hone sporting skills to the same extent as boys and men (Zipp \& Nauright, 2018). This practice attempts to integrate girls and women into patriarchal, hegemonic, and heteronormative frameworks (Forde \& Frisby, 2015). Further, this approach does not address systematic inequalities. As Forde and Frisby (2015, p. 890) contend: “. . . this (approach) is problematic if it simply involves girls and women adopting masculinized behaviors with little or no emphasis being placed on how masculinity is embedded in hegemonic power structures and how the behavior of some boys and men needs to change to promote safety and greater gender equality."

\section{Applying the Capability Approach to SFD}

\section{The CA in SFD Model}

In this study, we synthesize a critical feminist, GAD approach with the CA to develop a new theoretical framework for SFD. Using a gender lens, we contend that the CA can provide (a) a better understanding of how SFD influences the real opportunities (capabilities) of participants to live lives they value and (b) useful conceptual tools, including capabilities, conversion factors, functionings, and adaptive preference. This approach can help researchers and practitioners challenge neoliberal ideologies and support critical feminist perspectives, both of which scholars are calling for in the field of SFD (Darnell \& Dao, 2017; Hayhurst et al., 2015; Oxford \& Spaaij, 2019; Sanders, 2016).

The CA emerged primarily from the work Amartya Sen, an economist and philosopher from India (see Sen, 1992, 1999). The framework was built upon by Nussbaum $(2001,2011)$ and Robeyns (2003, 2005, 2006, 2017), among others. The CA includes the key elements of capabilities (or opportunities), the functionings (or outcomes) that flow from them along with the 
conversion factors that influence the capability set and the adaptive preferences that inform choices (Sen, 1999). At its best, the CA encourages a better understanding of how development initiatives are experienced, rather than restricting the focus of development (and development research) merely to prescribed outcomes, which can obscure underlying inequalities (e.g., gender, race, class), reinforce neoliberal ideologies and overlook restraints on peoples' freedoms (Robeyns, 2005). Robyens diagrammed her understanding of the $\mathrm{CA}$ in a dynamic model (Figure 1) to illustrate the relevance of multiple concerns to one's capability set and the selection of particular functionings $(2005,2017)$.

Applying the CA to SFD can help both researchers and practitioners to untangle some of the nuanced experiences of participants. The CA helps to move beyond evaluating if participants experience some form of (predetermined, measurable) personal development, and is more attuned to how these interventions are experienced. A focus on the how of development gives room for understanding how gender is experienced in SFD and allows for gender to be woven through the key elements of the CA framework (Zipp, 2017a). This focus on processes and possibilities over outcomes and outputs is a distinct departure from most SFD research, which is centered on the monitoring and evaluation strategies of practitioner organizations and/or funders. To expand on the concepts of the CA and apply it to SFD research and practice, we present this diagrammatic model. The model here is drawn directly from Zipp's doctoral dissertation (2017a), which was developed as an adaptation of Robeyns' CA model (Figure 1) to the field of SFD.

This model is unique among CA applications within SFD, as it applies a gender lens explicitly across all levels of conversion factors and adaptive preferences. We have provided sample capability sets in the model presented, but this model is flexible and designed for researchers and practitioners to substitute, edit, add, or remove capability sets in line with local contexts and program goals. In other words, this CA in SFD model provides the conceptual architecture to consider contextually determined capabilities and conversion factors. The capability set referenced in the model above is illustrative of the capabilities that are common to SFD programs. This model also organizes conversion factors, following (Robeyns, 2017), into personal, social, and environmental categories. Although the authors and other capability scholars recognize the complexity and interdependence of these factors, they are conceptually divided into these three categories to better unpack how capabilities are expanded or constrained based on contextual factors. It is important to note that the conversion factors presented here are examples to consider, not exhaustive lists. In providing these elements, we acknowledge a risk of missing other important aspects or overemphasizing normalized life experiences. However, we feel that the specificity of this model is valuable because it provides the aforementioned gender lens and prompts users to consider macrolevel environmental factors that are often under analyzed.

Capabilities are at the heart of this approach and can be defined as the full breadth of options, or real opportunities, that a person has. According to Sen (1999), the political or programmatic commitment to supporting capabilities, therefore, requires both the elimination of coercion or interference (negative freedom) and the promotion of real opportunities (positive freedom). In essence, the notion of capabilities, and capability expansion, in the context of development pushes for greater equality of people's actual opportunities to achieve, not only by removing interference, but also by enhancing people's abilities to convert resources into attainments or successes. Capabilities are what people are "able to be and do" (Robeyns, 2017, p. 38). They are generated as people engage with resources, using them in a way that is mitigated by various conversion factors (personal, social, and environmental). So, to best explain the CA, we move generally from left to right across the diagrammatic model. Along the way, we explain the key concepts of the CA, drawing from examples in development studies and SFD to demonstrate how the proposed model can contribute to research and practice.

Ingrid Robeyns (2017) provides the example of riding a bicycle to illustrate the concept of capabilities, conversion factors,

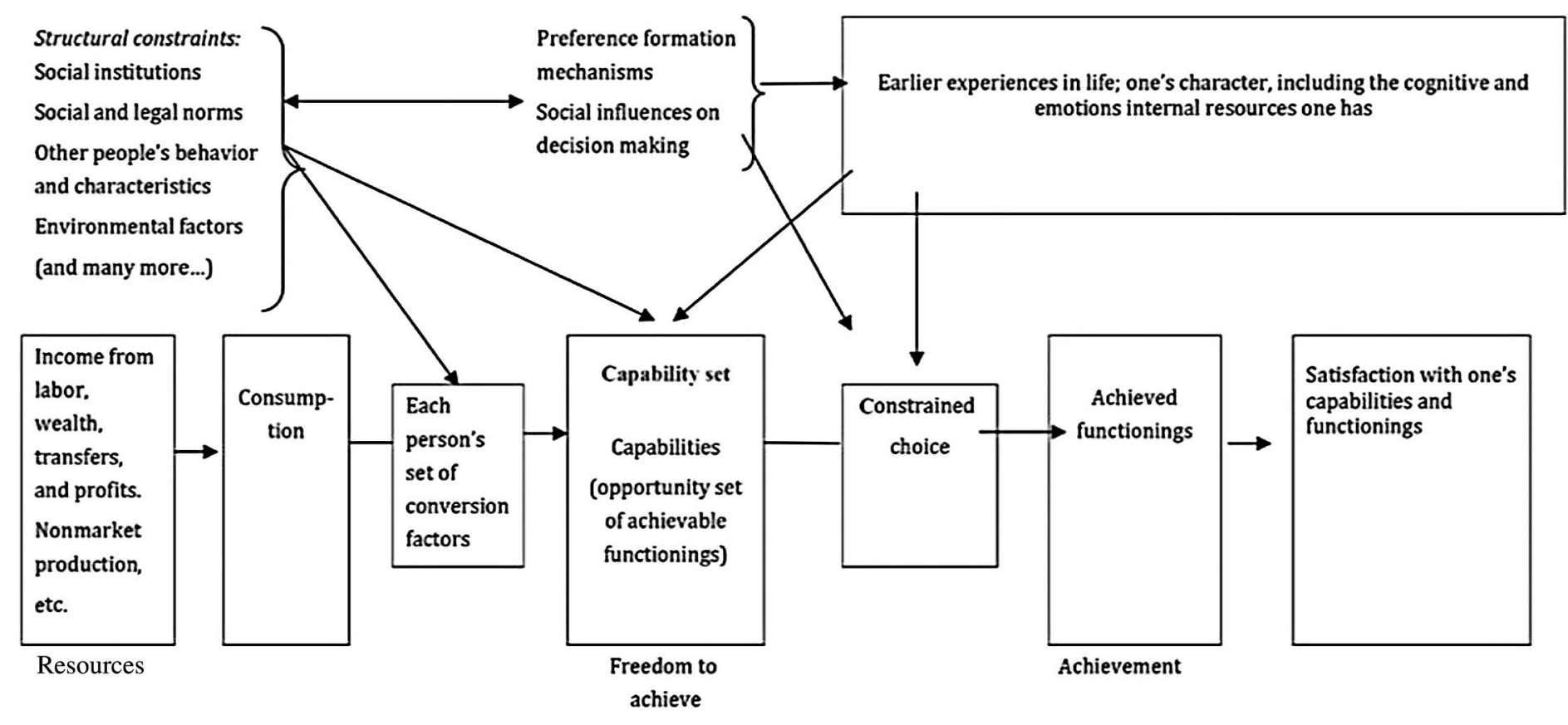

Figure 1 - Robeyns' (2017) model. 
and how the CA model works dynamically. In her example, for a person to achieve the mobility and exhilaration that riding a bicycle provides, various resources and conversion factors at the personal, social, and environmental levels must support this capability. First, she must have the required resources for bicycling. Obviously, access to a bicycle is necessary. Furthermore, access to an instructor, coach, or mentor of some kind to help her learn to ride is important. These resources, however, are interdependent on various conversion factors. For example, access to a coach or mentor is often mitigated by traditional gender norms (macrolevel environmental conversion factor), which could prevent her developing the physical ability to ride (personal conversion factor). Second, family and community members must not restrict, and may need to actively support, her decision to ride a bicycle, perhaps serving as mentors or role models (mesolevel social conversion factor). Finally, she must also be free from social and legal forces that would prevent her from riding, such as gender roles or religious laws (macrolevel social conversion factor), and live in an environment where riding is safe and accessible (e.g., bicycle paths or usable roadways [environmental conversion factor]). These factors are often inextricably interdependent and deeply gendered. Thus, they combine to and mitigate or promote one's real opportunity to ride a bicycle.

To explain the model and how it can be applied, the following sections will explore the key elements of the model, providing examples of how a critical feminist perspective of the CA can be an insightful and practical tool in SFD.

\section{Resources and Conversion Factors}

To provide an example of how the CA model can work in SFD, we expand on the example of menstruation. Recent studies show that many adolescent girls struggle with sport participation once they begin menstruating (Betty for Schools, 2018; Tingle \& Vora, 2018; Women in Sport, 2018; Zipp \& Standing, 2018). This is a very difficult topic, given the taboo nature of menstruation in most cultures, and particularly in cultures where rigid gender roles are enforced. This singular issue is present in resources and as a conversion factor at all three levels: personal, social, and environmental.

From a resource standpoint, access to private, clean, and safe toilet facilities for changing during periods is a key factor for schools and communities (Forde, 2009; World Health Organization, 2016). Although these resources are specific to local contexts, they are often determined by broader macro, environmental forces such as infrastructure, health regulations, and government policy (see Robeyns 2017 , chapter 2.12 for a more detailed explanation of how conversion factors and resources interact via generation of capability sets). Other resources, such as female coaches, may also have a profound impact on a person's experience of menstruation.

At a personal level, whether or not a girl has begun her menses is paramount to her experience in SFD. Around the world, the median age for one's first menstruation is 12 years old, the same age girls often transition to secondary school and a common time when they drop out of sport (Tingle \& Vora, 2018). Symptoms of menstrual periods vary, but can include cramping, fatigue, and headaches that may dissuade adolescent girls from participating in sport, despite growing evidence that exercise can ease these symptoms (Women in Sport, 2018).

At the social level, influences from family, school, and community are critical. Family socioeconomic status can cripple a girl's access to sufficient menstrual products (pads, cups, etc.) that can help them cope with their period bleeding. Such "period poverty" or lack of resources to obtain adequate menstrual products is a growing concern in international development (Bobel, 2018; Zipp $\&$ Standing, 2018). In sport and physical activity, these products are very important because many girls fear embarrassing leaks during rigorous movements such as running and jumping (Forde, 2009; Women in Sport, 2018). At school, education on menstrual health is often limited, laden with social restrictions, or missing altogether, leaving young girls unaware, unprepared, and embarrassed to talk about their experiences (Bobel, 2018).

On a macro level, deeply rooted stigma and myths regarding menstruation often create strict gender roles that socially marginalize girls and women. Evaluations from two SFD programs that delivered menstrual health education, Moving the Goalposts (Kenya) and the Naz Foundation (India), found that participants felt restricted by menstrual myths and felt empowered through learning sessions through their programs (Forde, 2009; Naz Foundation, 2017). "I no longer believe the myths society peddles about menstruation ..." claimed a 14-year-old Naz participant (Naz Foundation, 2017, p. 17). In her recent book, Bobel (2018) critiques the development sector, particularly the menstrual hygiene management movement, for overlooking or deemphasizing the menstruation stigma. Broad environmental factors such as education, health, infrastructure, and employment policies can have a profound impact on the experience of people who menstruate (Bobel, 2018).

The issue of menstruation illustrates the potential of this CA in SFD model. Researchers and practitioners should, therefore, recognize that adolescent girls may be experiencing dramatic physical changes that complicate their participation in sport and physical activity. Programs should consider participants' access to menstrual health education, resources for period care (e.g., menstrual products), and suitable toilet facilities to better support their participants. We have included puberty and menstruation among the conversion factors and toilet facilities among the resources in the model (Figure 2) to prompt researchers and practitioners to consider this issue. As demonstrated above, once one begins to unpack the experience of menstruation, it is clear that its reach extends beyond the personal level. The CA model includes other conversion factors commonly relevant within development studies, drawn from Robeyns' (2017) CA model and youth development theories (Cunningham \& Correia, 2003) that often underpin research and practice in SFD, which is largely targeted at children and youth. This model also layers in sport-relevant conversion factors, such as including coaches and SFD facilitators within community mentors. By examining the conversion factors through a gendered lens, we see that the overall experience, and capability, for girls and women to participate in sport is mitigated at various stages and levels by this oft-overlooked aspect of one's lived experience.

\section{Capabilities}

Focusing in on the concept of capabilities within the proposed SFD model, we can draw similar analogies to Robeyns' bicycle. Although many girls and women may seemingly have the "opportunity" to participate, a myriad of obscured or misunderstood gender-related conversion factors may restrict or eliminate it, thus adding nuance to our understanding of capability as real opportunity. Although these types of restrictions are included in much of the critical feminist research in SFD, the proposed CA model can help bring these concerns to the fore, supporting researchers and practitioners to better understand girls' and women's experiences. 


\section{Conversion Factors}

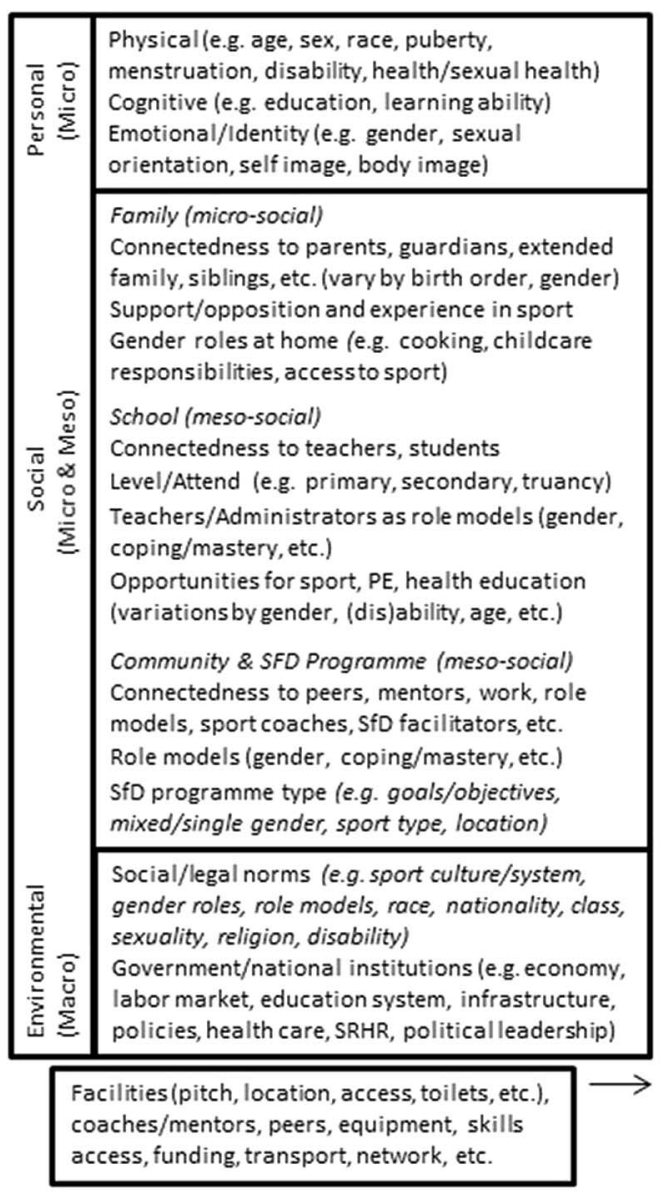

Resources

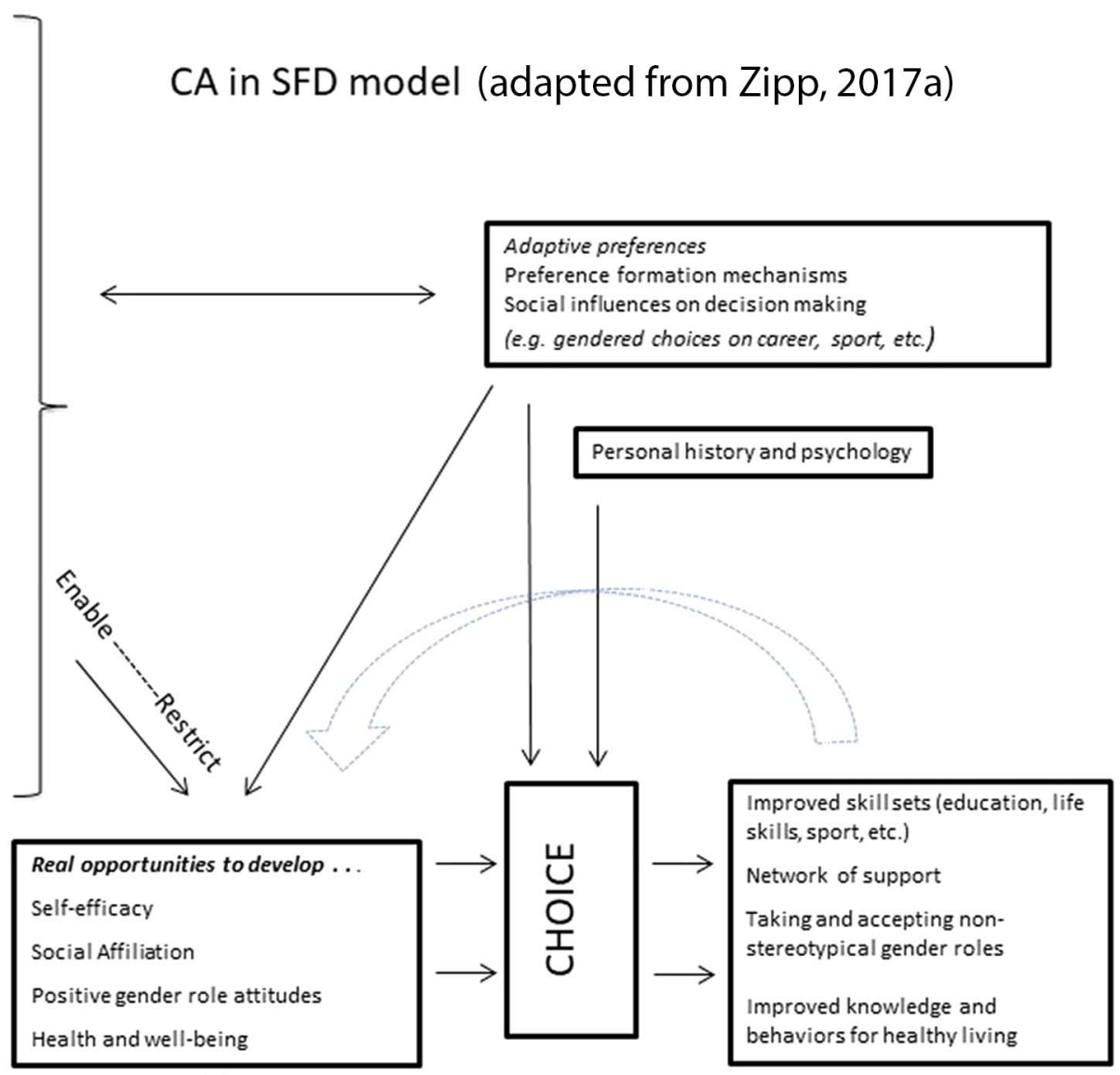

Capability Set

(Ideal) Achieved Functionings

\{Example of common Resources, Capability Set and Achieved Functionings in SfD. Individual programmes would insert/replace with own elements.\}

Figure 2 - Capabilities approach (CA) in sport for development (SFD) model. Adapted from Zipp's dissertation (2017a).

The illustrative model presented here includes four capabilities: self-efficacy, social affiliation, positive gender role attitudes, and health and well-being. These four capabilities represent common goals or objectives within the SFD movement. They are intended here as illustrative examples to be modified or replaced with contextually determined, relevant capabilities, along with specific resources and (ideal) achieved functionings. Among the capabilities presented here, "positive gender role attitudes" is clearly a gendered item. "Health and well-being" have fairly clear gender connotations, which are reinforced in the model by related conversion factors such as puberty, menstruation, sexual health, health education opportunities by gender, sexual and reproductive health rights, etc. Beyond these capabilities, we contend that the gender lens explicitly taken up in the analysis of conversion factors will help researchers and practitioners critically analyze how gender interacts with other capabilities less obviously gendered.

For example, "connectedness" is a social conversion factor that informs the capability development of "social affiliation" in our proposed model. Connectedness is a term borrowed from youth development studies, where scholars have found that close relationships to family, school, and community often serve as protective factors for positive youth development (Cunningham \& Correia, 2003). The model presented here accounts for gender in points of connectedness within the family, school, and community contexts (e.g., gender within family, gender differences in physical education at school, gender of role models/coaches). Studies in SFD have shown that the lack of female role models, coaches, and facilitators with SFD programs may restrict girls and women from more fully engaging as participants and reinforce negative gender stereotypes (Meier, 2015; Zipp \& Nauright, 2018).

Another example is self-efficacy, which is widely defined as the belief in one's ability to influence events in his or her life toward a specific outcome (Bandura, 1997). SFD proponents suggest that sport is an ideal format for building self-efficacy because a person can tangibly experience outcomes from her effort. For example, a participant who practices throwing, catching, kicking, running, or batting skills can see progress over time, supporting the key concept of self-efficacy, that she can influence an outcome in her life through her own effort (Coalter \& Taylor, 2010). Selfefficacy is distinct from its cousin, self-esteem in a very specific way. Although self-esteem refers to one's perceived worth or value as a whole, self-efficacy narrows in on the specific belief in one's ability to achieve or attain an outcome. This distinction positions self-efficacy nicely within the CA as it is implicated in the model's movement from capabilities to functionings (or achievements), the "doings" in Sen's framework. Self-efficacy also measures 
perceptions and beliefs, not actualized outcomes or functionings. Therefore, the focus is on the capability of believing in one's abilities, rather than the end product.

At first glance, self-efficacy may seem a rather gender-neutral concept. However, studies in sport and SFD have shown that gender plays a role (Spence et al., 2016; Zipp \& Nauright, 2018). In these studies, adolescent boys had higher levels of self-efficacy as related to physical activity or sport than adolescent girls. Boys' previous experience in sport, often through fathers or male family members, may better position them to enhance their sport skills and report higher self-efficacy than their girl counterparts. In essence, the social conditioning and exposure to sport varies by gender, thereby directing a person's interests, preferences, and decision making about sport. To further explore this aspect of gender and capability development, we explore the concept of adaptive preferences.

\section{Adaptive Preferences}

Another critical concept of the CA is adaptive preferences. Sen's work in development economics shows that women, as well as other members of marginalized groups or communities, often experience a form of normalized inequality that may affect their actual ability to achieve certain functionings. In this sense, women (or lesbian, gay, bisexual, transgender, queer communities, or people of color [LGBTQ]) who experience systemic oppression often become accustomed to selecting from a limited set of opportunities. An adaptive preference occurs when a person's subjective assessment of their own well-being is influenced by the objective reality of their lived experiences (Sen, 1999).

In other words, people who experience multiple axes of oppression are often systemically conditioned to select from a limited array of options; as a result, the CA draws attention to the importance understanding adaptive preferences. Although this aspect of the CA (like several others) is open to accusations of paternalism, by presuming that individuals do not recognize the limits or constraints of their own preferences, the implications are profound when deployed in a nuanced manner. The key point is that when women work to "formulate agency goals, the aspirations expressed are conditioned by their socioeconomic background and experience" (Burchardt, 2009, p. 13). In this sense, the act of choosing functionings from an array of capabilities is necessarily influenced by structures and experiences of inequality. More specifically, gendered socialization often has an unseen effect on the capabilities of women (and other marginalized groups) by limiting their recognition of, and aspirations for, certain capabilities and functionings.

This point, in turn, has specific implications for sport, given the gendered socialization in and of sport participation. Sen (1999), Nussbaum (2011), and others (e.g., Robeyns, 2017) have argued for the importance of considering adaptive preferences in the CAparticularly with respect to girls and women. Sport is so deeply entrenched in binary modes of gender and is constructed in a way that privileges males and traditional aspects of masculinity (heteronormativity). Participants in an all-girls SFD program in St. Lucia summed up this binary nicely when they explained that: "Getting muscles is good for men, but not for girls," (Zipp, 2017b, p. 1927). By examining how preferences are formed and adapted according to gender, SFD scholars, and practitioners can use the CA to consider the processes and practices of how sport is experienced in development programming. This approach helps to avoid too narrow a focus on outcomes. For example, research on the impacts of Title IX legislation in the United States have shown that the expansion and promotion of opportunities for girls to play sport has increased girls' interest in playing sport (Acosta \& Carpenter, 2014; Ware, 2014). Title IX demonstrates the "if you build it, they will come" approach to inclusivity, which reflects the power of adaptive preferences.

In SFD, specifically, gendered sport roles have been discussed in research from feminist scholars for years (Chawansky, 2011; Kidd, 2013; Knoppers \& McDonald, 2010; Meier, 2005). However, the concept of adaptive preferences and preference formation has only recently become part of the conversation. In their article on SFD in the West Indies, Zipp and Nauright (2018) show that girls tend to adapt to rigid social roles that push them into certain sports and away from others, or out of sport altogether. In the West Indies, cricket is king; the British colonial legacy sport has long been a source of national pride. Although the women's regional team has achieved international success in recent years, the men's game is omnipresent, with statues and roundabouts named after hometown legends. In this environment, "a game such as cricket is passed down from father to son for generations, but daughters are rarely invited to play" (Zipp \& Nauright, 2018, p. 33).

The male-dominated legacy of the game translates to boys with more experience and encouragement to play, which helps them build skill and confidence in their cricket abilities (self-efficacy). What happens when they are then mixed with girls in SFD programs? In one case, at the Sport for Life program in Barbados, the girls left a cricket drill to go play separately, turning cartwheels on the sidelines of the pitch. They literally moved to the margins of the game that they felt unwelcome in and created their own space to play. This incident reflects the adaptive preferences of boys and girls in the program, with boys socialized to prefer cricket and girls, lacking the socialization and practice at the game, preferring to find their own way. Likewise, boys experience constrained choice in sport under similar social influences (e.g., boys do not play netball; Zipp \& Nauright, 2018). Sen's adaptive preferences help clarify why people make the gendered choices they make in sport and SFD.

What can program designers and researchers learn from this story? Mixed gender sport is often seen as the answer to gender equality - just include girls and women in the game and they will benefit. But this is an outdated GID approach. Researchers and practitioners might not question that girls lack experience in cricket, do not prefer to play it over other sports or may not want to play it with boys. In contrast, the critical feminist lens requires researchers to engage with this issue from a more comprehensive standpoint. In this light, the potential of a gendered lens in the CA and SFD incorporates gender into the analysis across all phases and levels (through the influence of conversion factors) in a more effective GAD approach.

Adaptive preferences work in varied ways and can help explain the phenomenon of girls and women's rugby flourishing in Laos and Vietnam. Through an innovative SFD program, funded by the ChildFund's "Pass it Back" initiative and administered through the Women Win Foundation, rugby has become a sport of choice among female participants (Owens, 2016). More than half of the Pass it Back rugby participants are female, despite struggles to reach female participants in previous attempts with other sports. Why did rugby succeed where other sports failed? Essentially, other sports were considered too masculine. Rugby was an unknown sport, without preconceived gender restrictions, despite its aggressive and physical play (Johnston, 2015). The rejection of other mainstream sports as too masculine and acceptance of rugby as a sport for girls and women reflects the power of adaptive preferences. The preference formation of female participants for other sports was 
determined (e.g., football is not for girls and women), but they had no preferences regarding rugby, allowing them the freedom to play.

Considering both examples, cricket in the West Indies and rugby in southeast Asia, adaptive preferences help clarify the gender lens of the CA model. This encompassing gender lens, built on elements of the CA framework, applies across capabilities that do not explicitly deal with gender (e.g., social affiliation, selfefficacy) as well as those that do (e.g., positive gender role attitudes, body image), illuminating interdependencies and intersectionalities that are often overlooked. This model can provide both academics and practitioners practical tools and language (e.g., adaptive preferences) for designing programs and evaluations with these influences in mind. The emphasis on broader social and community influences also encourages the larger SFD movement of a responsibility to address, advocate, and challenge the restrictive gender roles that systematically subjugate their female participants and restrict the type of masculinity available to male participants.

In sum, we draw attention here to the fact that Sen (1999), Robeyns (2006), and Nussbaum (2011) have all argued that adaptive preferences should be considered when using the CA, which in turn constitutes one of the main arguments for moving beyond assessments of development results and toward a multidimensional account of impact and equality within the processes of development, such as they are. Adaptive preferences provide a key conceptual contribution for SFD toward understanding several ways that SFD programming and interventions are implicated in processes of social change.

\section{Conclusions and Recommendations}

\section{The Capability Approach in Sport for Development}

In broad strokes, the CA has been used in sport studies as a theoretical framework to explore, explain, and promote positive social change (if not necessarily development). Jarvie and Sikes (2012), for example, drew on Sen's (1992, 1999) comments regarding inequality to suggest that a capability lens may provide insight into the role that sport, and sporting experiences, have in the broader international development context. In turn, and more recently, the CA has been applied to the subfield of SFD research, which is arguably more specific than social change and characterized by the intentional organization and mobilization of sport to meet development goals like gender empowerment, economic growth, and health promotion. This growing literature has illustrated a large measure of compatibility and applicability between the CA and SFD. In particular, Sen's work has led to critical consideration of sport's (in)ability to address structural development inequalities while echoing, for example, Hartmann and Kwauk's (2011) "transformative vision" for SFD. In this way, the potential for the CA to advance SFD theory and practice beyond the limited study of SFD's effects and processes of monitoring and evaluation, has become increasingly clear.

Indeed, Darnell and Dao (2017) concluded that the CA can "contribute toward an ethical conceptualization of sport's place within efforts to improve the lives of underserved people and communities," (p. 24) by arguing that the ethical and political underpinnings of the CA are congruent with a transformative vision for SFD. In so doing, they highlight Nussbaum's (2011) central capabilities list and show that the CA offers an opportunity to advance sport as both intrinsically and instrumentally valuable, and therefore a right to which people are entitled. In a compatible analysis, Svensson and Levine (2017), drawing on Sen, suggest that the CA encourages, and even forces, practitioners and researchers to consider the implications of institutional and policy arrangements in SFD and how these impact the freedoms and capabilities of participants (Svensson \& Levine, 2017). They further suggest that the CA in SFD research might provide a normative guideline for research without requiring a commitment to particular epistemological or methodological approach.

In practice, reified gender differences must be integrated into a comprehensive analysis in the CA paradigm. Adaptive preferences have a fundamental effect on a person's choice and experience of achieved functionings; to facilitate a move from obscured input in a traditional theory of change model to an informative insight that contributes to understanding processes of development, adaptive preference appears in Robeyns' model as an element of constrained choice. Thus, functionings (as outcomes in/of SFD) are still a part of analysis in the CA framework, but they are deemphasized in favor of more thorough exploration of the process by which capabilities are generated and experienced. By considering conversion factors (including as a contributor to preference formation), the CA reminds researchers and practitioners that SFD does not work in isolation, but rather within larger systems and structures that are often in neoliberal, postcolonial, and patriarchal frames. Much of the SFD research and program evaluation tends to overlook or deemphasize these social and environmental factors (Sanders, 2016). Not only does examining SFD from this vantage help explain and understand the experiences of participants and practitioners in SFD, but it also helps the researcher form effective critiques.

A critical capability analysis of SFD, especially with an overt concern with gender, shows the importance of supplementing the $\mathrm{CA}$ with other theoretical frameworks. The CA provides the framework for a variety of contextually appropriate theories to be integrated into a comprehensive understanding of experiences in and of development. This includes, but is not limited to, feminist theory, queer theory, postcolonial theory, critical race theory, youth development theory, social capital frameworks, and myriad others. Applying the CA to SFD is, inter alia, an attempt to better capture the interplay between social context and experiences and processes of development by providing the conceptual architecture to integrate these other theoretical frameworks. Robeyns' model represents a compelling departure point for feminist analyses and critique of SFD for exactly this reason; it requires researchers and practitioners to contend with the complexity of personal, social, and environmental conversion factors that both enable and constrain the individuals that constitute social arrangements. In this way, it provides a platform to engage with modes of oppression as they manifest in the lives of individuals - by limiting their real opportunities to live lives that they have reason to value.

Altogether, it is clear that gender, equality, and capability are inextricably related, and, thus, should not be compartmentalized. The outmoded GID approach, common in SFD, falls short of engaging critically with the lived experience of participants. A more transformative, GAD approach is needed to protect, promote, and expand capabilities as well as to avoid isolating girls and women's "issues" as distinct from overall program purpose. SFD is shaped by the gendered, lived experiences of program participants and communities where SFD activities are interwoven with social norms, structural constraints, and complex histories. Further, the conceptual tools inherent in the $\mathrm{CA}$, such as capabilities, conversion factors, and adaptive preference, can equip SFD with much more comprehensive, multidimensional methods of evaluationincluding those that engage by necessity with systemic oppression. 
The CA framework presented in this article and applied in the conceptual model, is one way in which the CA can be employed to support research and applied practice in SFD. The flexible CA paradigm can be adapted and applied in various ways, drawing on the key elements of capabilities, conversion factors, adaptive preferences, and functionings.

\section{Limitations}

Of course, we must consider the limitations, weaknesses, and risks of any approach. The CA has been criticized as supporting neoliberal institutions and ideologies. We have made the case for using the CA to challenge these norms, but applying the model is much trickier in practice. It is clearly possible that both practitioners and researchers might include neoliberal assumptions, bias and misunderstandings into the model, thus reinforcing the very issues of inequality and injustice that it seeks to challenge.

Another concern is identifying the difference between resources, capabilities, and functionings. This process can be unclear and subjective. For example, the differences between social affiliation (capability set) and a network of support (functioning) are ambiguous. If an SFD participant develops positive social relationships within/outwith the program, how is this translated into a supportive network? If those relationships are with people who have little power or influence on the participant's surrounding environment, the network may not be able to provide any support or very limited support.

We also recognize the reality of research and nongovernmental organization program funding. Outcomes, outputs, and measurable achievements, such as achieved functionings, are valuable arguments for program and research funding. This type of explanatory research approach, with a focus on process over outcomes, can make it more difficult to demonstrate the kind of development impact that funders often seek.

It is difficult for any model to address all of these possible issues and concerns, and this CA in SFD model is susceptible to these weaknesses. In particular, it is a broad application of the CA and must be localized within context. This CA model strives to create a framework for localization, specifically by including the various levels of conversion factors to prompt researchers and program designers to consider these influences. However, by providing more specific examples to contextualize the model to SFD, we may be reinforcing normalized aspects of participants' lives (e.g., school and family may not apply to out of school orphans). Along those same lines, there are concerns in SFD that many researchers and nongovernmental organizations from wealthy, Global North countries are conducting work with limited input from local people (Banda \& Holmes, 2017). This model cannot prevent such an approach.

\section{Recommendations and Future Research}

To effectively engage with this model, a localized approach, built from the subaltern voices of those from the within the communities targeted by SFD, particularly from the Global South, must be applied. The newly released book, Localizing Global Sport for Development, makes the case for localizing SFD and provides insight on challenging the neoliberal approaches that have defined much of the SFD movement (Lindsey, Kay, Banda, \& Jeanes, 2017). In collaboration with our CA model, this localized perspective can help researchers and practitioners address the (often hidden) barriers to and stimulators of capability development. For example, to address the above-mentioned issue of menstruation, future research and practice should seek to understand the impact of menstruation on participants, concerns of period poverty, and advocate for health and education policy that supports menstrual health.

Future research should also build from understanding macrolevel environment conversion factors on SFD toward advocating for policies and social change that challenges the inequalities and injustices that systematically oppress, restrict, and subjugate people. The SFD movement has matured to the point that advocacy should be a central tenant to program design and research (Sanders, 2016). Those who work and research SFD are generally drawn to this field because they want to make a positive impact. Advocacy on public policy and using sport as a highly visible platform to promote social change is an important and effective route to making that impact. We believe that this CA approach can support those researchers and practitioners to collaborate, with a clearer understanding of the complexities of gender dynamics in sport, toward a more impactful, inclusive, and sustainable SFD movement.

\section{Notes}

'The model presented is drawn directly from the lead author's doctoral dissertation. The dissertation will be cited as (Zipp, 2017a) for the purpose of this blind review.

${ }^{2}$ For practical purposes, we will rely on binary gender identity terms such as girls/boys, men/women, and female/male in this article. We acknowledge, however, that gender is nonbinary and seek to use more inclusive terms such as "all genders" as much as possible.

\section{References}

Acosta, R.V., \& Carpenter, L.J. (2014). Women in intercollegiate sport: A longitudinal national study thirty-seven year update (1977-2014). Retrieved from http://www.acostacarpenter.org/2014\%20Status\% 20of\%20Women $\% 20$ in $\% 20$ Intercollegiate $\% 20$ Sport $\% 20-37 \% 20$ Year \%20Update\%20-\%201977-2014\%20.pdf

Banda, D., \& Holmes, M. (2017). Sport-for-development and the struggle for subaltern voices to be recognised: A response to Manley, Morgan and Atkinson. International Journal of Sport Policy and Politics, 9(4), 723-738. doi:10.1080/19406940.2017.1372794

Bandura, A. (1997). Self-efficacy: The exercise of control. New York, NY: W.H. Freeman.

Betty for Schools. (2018). Period taboos prevent girls from participating in sport at school. Retrieved from https://bettyforschools.co.uk/news/ period-taboo-research

Bobel, C. (2018). The managed body: Developing girls and menstrual health in the global south. Basingstoke, UK: Palgrave Macmillan.

Burchardt, T. (2009). Agency goals, adaptation and capability sets. Journal of Human Development and Capabilities, 10(1), 3-19. doi:10.1080/ 14649880802675044

Carney, A., \& Chawansky, M. (2016). Taking sex off the sidelines: Challenging heteronormativity within "sport in development" research. International Review for the Sociology of Sport, 51(3), 284-298. doi:10.1177/1012690214521616

Chawansky, M. (2011). New social movements, old gender games?: Locating girls in the sport for development and peace movement. Research in Social Movements, Conflicts and Change, 32, 123-136.

Chawansky, M., \& Hayhurst, L.M.C. (2015). Girls, international development and the politics of sport: Introduction. Sport in Society, 18(8), 877-881. doi:10.1080/17430437.2014.997587 
Coalter, F., \& Taylor, J. (2010). Sport-for-development impact study: A research initiative funded by comic relief and UK sport and managed by international development through sport. Comic Relief, UK Sport Department of Sports Studies, University of Stirling.

Cunningham, W., \& Correia, M. (2003). Caribbean youth development: Issues and policy directions (English). Washington, D.C: World Bank. Retrieved from https://elibrary.worldbank.org/doi/pdf/10. 1596/0-8213-5518-X

Darnell, S.C. (2007). Playing with race: Right to play and the production of whiteness in "development through sport." Sport in Society, 10(4), 560-579. doi:10.1080/17430430701388756

Darnell, S.C. (2012). Sport for development and peace: A critical sociology. London, UK: Bloomsbury Academic.

Darnell, S.C., \& Dao, M. (2017). Considering sport for development and peace through the capabilities approach. Third World Thematics: A TWQ Journal, 2(1), 23-36. doi:10.1080/23802014. 2017.1314772

Forde, S. (2009). Playing by their rules: Coastal teenage girls in Kenya on life, love and football. Charleston, SC: Create Space.

Forde, S.D., \& Frisby, W. (2015). Just be empowered: How girls are represented in a sport for development and peace HIV/AIDS prevention manual. Sport in Society, 18(8), 882-894. doi:10.1080/ 17430437.2014 .997579

Harrison, C. (2018). How is menstrual hygiene management incorporated into sport for development programmes in the global south (Unpublished Master's Thesis). University of Stirling, Stirling, United Kingdom.

Hartmann, D., \& Kwauk, C. (2011). Sport and development: An overview, critique, and reconstruction. Journal of Sport \& Social Issues, 35(3), 284-305. doi:10.1177/0193723511416986

Hayhurst, L.M.C. (2011). Corporatising sport, gender and development: Postcolonial IR feminisms, transnational private governance and global corporate social engagement. Third World Quarterly, 32(3), 531-549. doi:10.1080/01436597.2011.573944

Hayhurst, L.M.C., Kay, T., \& Chawansky, M. (Eds.). (2015). Beyond sport for development and peace: Transnational perspectives on theory, policy and practice. Oxon, UK: Routledge.

Jarvie, G., \& Sikes, M. (2012). Running as a resource of hope? Voices from Eldoret. Review of African Political Economy, 39(134), 629-644. doi:10.1080/03056244.2012.738416

Johnston, P. (2015, June 6). In Laos, women's rugby flourishes on unsuitable grounds. Reuters. Retrieved from https://uri.libguides. com/cite/apa6th

Kidd, B. (2013). Sports and masculinity. Sport in Society, 16(4), 553-564. doi:10.1080/17430437.2013.785757

Knoppers, A., \& Anthonissen, A. (2008). Gendered managerial discourses in sport organizations: Multiplicity and complexity. Sex Roles, 58, 93-103. doi:10.1007/s11199-007-9324-z

Knoppers, A., \& McDonald, M. (2010). Scholarship on gender and sport in sex roles and beyond. Sex Roles, 63 311-323. PubMed ID: 20835388 doi:10.1007/s11199-010-9841-z

Lindsey, I., Kay, T., Banda, D., \& Jeanes, R. (2017). Localizing global sport for development. Manchester, UK: Manchester University Press.

McDonald, M.G. (2015). Imagining neoliberal feminisms? Thinking critically about the US diplomacy campaign, "Empowering women and girls through sports." Sport in Society, 18(8), 909-922. doi:10. 1080/17430437.2014.997580

Meier, M. (2005). Gender equity, sport and development. Biel/Bienne: Swiss Academy for Development.

Meier, M. (2015). The value of female sporting role models. Sport in Society, 18(8), 968-982. doi:10.1080/17430437.2014.997581
Naz Foundation. (2017). Young people's initiative: Menstruation session and data (Unpublished Report), New Delhi.

Nussbaum, M.C. (2001). Symposium on Amartya Sen's philosophy: 5 Adaptive preferences and women's options. Economics and Philosophy, 17 67-88. doi:10.1017/S0266267101000153

Nussbaum, M.C. (2011). Creating capabilities: The human development approach. Cambridge: Harvard University Press.

Owens, J. (2016). "Pass It Back:" Leveraging the position of Rugby as a non-mainstream sport in Asia. sportanddev.org. Retrieved from https://www.sportanddev.org/en/article/news/pass-it-back-leveragingposition-rugby-non-mainstream-sport-asia

Oxford, S., \& McLachlan, F. (2018). "You have to play like a man, but still be a woman:" Young female Colombians negotiating gender through participation in a sport for development and peace (SDP) organization. Sociology of Sport Journal, 35 258-267. doi:10.1123/ssj.2017-0088

Oxford, S., \& Spaaij, R. (2019). Gender relations and sport for development in Colombia: A decolonial feminist analysis. Leisure Sciences, 41(1-2), 54-71. doi:10.1080/01490400.2018.1539679

Robeyns, I. (2003). Sen's capability approach and gender inequality: Selecting relevant capabilities. Feminist Economics, 9(2-3), 61-92. doi:10.1080/1354570022000078024

Robeyns, I. (2005). The capability approach: A theoretical survey. Journal of Human Development, 6(1), 93-117. doi:10.1080/ 146498805200034266

Robeyns, I. (2006). The capability approach in development. The Journal of Political Philosophy, 14(3), 351-376. doi:10.1111/j.1467-9760. 2006.00263.x

Robeyns, I. (2017). Wellbeing, freedom and social justice: The capability approach re-examined. Cambridge, UK: Open Book Publishers.

Saavedra, M. (2009). Dilemmas and opportunities in gender and sport-indevelopment. In R. Levermore, \& A. Beacom (Eds.), Sport and international development (pp. 124-155). New York, NY: Palgrave Macmillan.

Sanders, B. (2016). An own goal in sport for development: Time to change the playing field. Journal of Sport for Development, 4(6), 1-5.

Sen, A. (1992). Inequality reexamined. Oxford: Oxford University Press. Sen, A. (1999). Development as freedom. New York: Oxford University Press.

Spence, J.C., Blanchard, C.M., Clark, M., Plotnikoff, R.C., Storey, K.E., \& McCargar, L. (2016). The role of self-efficacy in explaining gender differences in physical activity among adolescents: A multilevel analysis. Journal of Physical Activity and Health, 7(2), 176-183. doi:10.1123/jpah.7.2.176

Suzuki, N. (2017). A capability approach to understanding sport for social inclusion: Agency, structure and organisations. Social Inclusion, 5(2), 150-158. doi:10.17645/si.v5i2.905

Svensson, P.G., \& Levine, J. (2017). Rethinking sport for development and peace: The capability approach. Sport in Society, 20(7), 905-923. doi:10.1080/17430437.2016.1269083

Thorpe, H., \& Chawansky, M. (2017). The gendered experiences of women staff and volunteers in sport for development organizations: The case of transmigrant workers of Skateistan. Journal of Sport Management, 31(6), 546-561. doi:10.1123/jsm.2017-0147

Tingle, C., \& Vora, S. (2018). Break the barriers: Girls' experiences of menstruation in the UK. Retrieved from https://plan-uk.org/file/planuk-break-the-barriers-report-032018pdf/download?token=Fs-HYP3v

Ware, S. (2014). IX: A brief history with documents. Longrove, IL: Waveland Press.

Women in Sport. (2018). Puberty \& sport: An invisible stage. The impact on girls' engagement in physical activity. Funded by Sport England. Retrieved from https://www.womeninsport.org/wp-content/uploads/ 2018/08/Puberty-PP.pdf 
World Health Organization. (2016). Core questions and indicators for monitoring WASH in schools in the sustainable development goals. UNICEF and World Health Organization. Retrieved from file://C:/Users/sz4/Desktop/BHAG/Menstrual\%20health\%20and\% 20MHM/WASH\%20JMP-2016-core-questions-and-indicators-formonitoring-WinS.pdf

Zipp, S.. (2017a). Changing the game or dropping the ball? : Sport as a human capability development for at risk youth in Barbados and St. Lucia (ISS PhD Theses). Erasmus University Rotterdam. Retrieved from https://www.stir.ac.uk/research/hub/publication/512727
Zipp, S. (2017b). Sport for development with "at risk" girls in St. Lucia. Sport in Society, 20(12), 1917-1931. doi:10.1080/17430437.2017. 1232443

Zipp, S., \& Nauright, J. (2018). Levelling the playing field: Human capability approach and lived realities for sport and gender in the West Indies. Journal of Sport for Development, 6(10), 38-50.

Zipp, S., \& Standing, K. (2018, May). Celtic FC leads way in tackling period poverty, now other clubs need to follow. The Conversation. Retrieved from https://theconversation.com/celtic-fc-leads-way-intackling-period-poverty-now-other-clubs-need-to-follow-96748 